\title{
BNIP3L Is a New Autophagy Related Prognostic Biomarker for Melanoma Patients Treated With AGI-101H
}

\author{
URSZULA KAZIMIERCZAK $^{1}$, TOMASZ KOLENDA ${ }^{1}$, DARIUSZ KOWALCZYK ${ }^{2}$, \\ JACEK MACKIEWICZ ${ }^{3,4}$ and ANDRZEJ MACKIEWICZ ${ }^{1,5}$ \\ ${ }^{1}$ Department of Cancer Immunology, Chair of Medical Biotechnology, \\ Poznan University of Medical Sciences, Poznan, Poland; \\ ${ }^{2}$ Department of Radiotherapy III at Greater Poland Cancer Centre, Poznan, Poland; \\ ${ }^{3}$ Department of Medical and Experimental Oncology, \\ University of Medical Sciences at Heliodor Swiecicki Clinical Hospital, Poznan, Poland; \\ ${ }^{4}$ Department of Biology and Environmental Sciences, \\ Poznan University of Medical Sciences, Center of Medical Biology, Poznan, Poland; \\ ${ }^{5}$ Department of Cancer Diagnostics and Immunology at Greater Poland Cancer Centre, Poznan, Poland
}

\begin{abstract}
Background/Aim: Skin melanoma belongs to the most invasive malignancies with no cure for a progressing disease. Personalized therapy would allow for the selection of patients that will benefit from treatment. For this purpose, proper predictive biomarkers must be defined. Materials and Methods: Allogeneic whole-cell gene-modified therapeutic melanoma vaccine (AGI-101H) was applied in advanced melanoma patients. Humoral responses were analyzed using SEREX, and in silico gene expression analysis in TCGA melanoma patients was performed. Results: A specific antibody response was raised against an antigen identified as BNIP3L, which correlated with a good prognosis. Moreover, AGI-101H directs an immune response against autophagy, as BNIP3L is a marker of this process. Medium and high expression of BNIP3L was also linked with longer overall survival. Conclusion: BNIP3L is a candidate prognostic marker of clinical outcome of melanoma patients treated with AGI-101H, and may be considered as a prediction marker for patient survival.
\end{abstract}

Cutaneous melanoma is responsible for $75 \%$ of skin cancerrelated deaths, and its incidence has been steadily increasing

This article is freely accessible online.

Correspondence to: Urszula Kazimierczak, Ph.D., Chair of Medical Biotechnology, Department of Cancer Immunology, Poznan University of Medical Sciences, 15 Garbary St., 61-866 Poznan, Poland. Tel: +48 618850665/+48 790470640, Fax: +48 618528502, e-mail: ukazimierczak@ump.edu.pl

Key Words: Melanoma, biomarker, BNIP3L, autophagy, melanoma vaccine. worldwide (1). Metastatic melanoma is highly resistant to conventional therapies. However, novel systemic therapy approaches such as targeted therapy with BRAF and MEK inhibitors (2) or various immunotherapy approaches have been exploited. These therapies include the adoptive transfer of immune cells (3), cancer vaccines, peptides, DNA, and the use of immune checkpoint inhibitors (ICI) such as anti-CTLA-4 or programmed death factor 1 (PD-1) monoclonal antibodies (4). The latter ones significantly improved the prognosis of melanoma patients; however, as monotherapy, they still demonstrate some limitations. Accordingly, combinatorial strategies are given the most attention nowadays. They include the combination of different ICI, ICI with cancer vaccines, kinase inhibitors etc. (5). However, the predictive and prognostic biomarkers are still required $(6,7)$.

The specific adaptive anti-cancer immune response comprises two phases- induction and effector. The induction phase can be activated and maintained by cancer vaccines. Moreover, in combined immunotherapy approaches, cancer vaccination generates tumor specific effector T cells. Since 1997, we have been investigating the therapeutic efficacy of whole cell melanoma vaccine (AGI-101H) in patients with advanced melanoma. AGI-101H (the first in its class) is an allogeneic tumor cell vaccine consisting of two human melanoma cell lines: Mich-1 and Mich-2, which were genetically modified to secrete designer cytokine Hyper-IL6 (H6), which serves as molecular adjuvant (8). H6 is a fusion protein of interleukin 6 (IL-6) and its soluble receptor (sIL6R, gp80). It directly targets gp130, a signal-transducing subunit, and activates the JAK1/STAT3-P/Oct4 pathway both in the paracrine and autocrine manners. Exposure of vaccine cells to H6 led to the conversion of their phenotype into melanoma stem cell (MSC) - like with high ALDH1H1 
expression (9). At the vaccination site, H6 stimulates allogeneic $\mathrm{T}$ cell response, inhibits $\mathrm{T}$ regulatory (Treg) cell formation, induces dendritic cell (DC) maturation, and presentation of cryptic antigens. It stimulates memory CD4+ and $\mathrm{CD} 8+\mathrm{T}$ cell formation, activates natural killer cells (NK), stimulates granulocyte-macrophage colony-stimulating factor (GM-CSF) secretion by lymphocytes (10), and decreases the number of myeloid-derived suppressor cells (MDSCs) (9). Down-stream H6 generates specific effector T cells (CTL) targeting melanoma differentiation antigens (MAGE-A, -A1, -A9, -A12, BAGE, GAGE-1, -2, -8, GAGE3, -4, -5, -6 -7, -7B, NY-ESO1, gp100, CTp11, PRAME, NA17A, TRP-1, TRP-2, Sox-10, SSX-1, HD-MM-05, -07,21, -22, and -25) (11) and stem cells antigen ALDH1A1. AGI101-H reduces myeloid-derived suppressor cells (MDSC) and regulatory T cells (Treg) in circulation (9).

Here, we analyzed the humoral immune response in 38 patients with stage III and IV melanoma treated with AGI$101 \mathrm{H}$. We employed a phage display technology and SEREX (Serological Analysis of Recombinant cDNA Expression Libraries). We found one peptide - BNIP3L (BCL-2/adenovirus E1B $19 \mathrm{kd}$-interacting protein-like) and enhanced production of anti-BNIP3L antibodies, which positively correlated with the clinical outcome of patients. Since BNIP3L is one of the critical factors regulating autophagy, it seems that AGI-101H is targeting the autophagy process as well. Accordingly, it may be proved to be a good prognostic factor for melanoma patients immunized with AGI-101H.

\section{Materials and Methods}

Patients and sera. This is a retrospective study using archived serum samples. The clinical studies were approved by the Local Ethical Committee (330A/96 dated 13 Sep 1996, 477A/97 dated 3 April 1997). All patients signed the informed consent form. The study was carried out in 38 patients participating in 2 clinical trials (A phase II trial: the evaluation of the efficacy and toxicity of an allogeneic melanoma vaccine, genetically modified, with interleukin 6/soluble interleukin 6 receptor complex (Hyper-IL-6), in patients with resected melanoma; A phase II trial: the evaluation of the efficacy and toxicity of an allogeneic melanoma vaccine, genetically modified, with interleukin 6/soluble interleukin 6 receptor complex (Hyper-IL-6), in patients with measurable melanoma metastases). The vaccine was administered 8 times in 2-week intervals (induction phase), and then ones per month (maintenance phase) until death. Twenty out of 38 (53\%) patients developed progression of the disease (PD), 10 out of $38(27 \%)$ responded to the treatment displaying either disease stabilization (SD), a partial response (PR), or a complete response (CR), and 8/38 patients received a vaccine in the adjuvant setting after the complete resection of melanoma. Sera used in the study were collected 6 months after the first dose of the vaccine administration and were stored in $-20^{\circ} \mathrm{C}$ until analyses.

Serological screening of recombinant cDNA expression libraries (SEREX). The immunological screen was performed as described previously (12). Briefly, sera were first diluted 1:10, pre-absorbed with $E$. coli and phage proteins. Pre-absorbed sera were then diluted 1:100 (final dilution) and used in screening. cDNA phage library derived from melanoma was used for serological identification of antigens by recombinant expression cloning. E. coli was transduced with recombinant lambda-ZAP phages and plated onto NZY-agar plates. Resultant plaque proteins were subsequently blotted into nitrocellulose membranes, which were then incubated with sera. Each serum was screened with approximately $2.5 \times 10^{4}$ bacterial clones expressing different genes from a melanoma gene library, in at least three separate experiments Reactive antibodies were detected with alkaline phosphatase-coupled secondary anti-human IgG antibody and visualized with 5-bromo-4-chloro-3-indolyl phosphate (BCIP) and nitroblue tetrazolinum (NBT). Positive monoclonal phages were subjected to in vivo excision of the pBluescript phagemids allowing the inserts to be analyzed by sequencing. A secondary SEREX was then performed with all of the other sera.

Sequence analysis. Sequencing was performed using the ABI Prism Genetic Analyser and Big Dye Terminator Cycle Sequencing Ready Reaction Kit with the primers recognizing the $\mathrm{T} 3$ and $\mathrm{T} 7$ promoters of the pBluescript phagemid. The homologous sequences were searched with a blast algorithm using the HUSAR package from the Biocomputing Service Group at the German Cancer Research Center.

TCGA data. The TCGA expression data of BNIP3L, expression of selected genes, and clinical data were downloaded from cBioPortal (Skin Cutaneous Melanoma, TCGA, Provisional, 479 samples) (13), from the UALCAN databases (14), and from StarBase v3.0 (15) for 472 cancers. All data is available online; access is unrestricted and does not require patient consent or other permissions. The use of the data does not violate the rights of any person or any institution.

Data analysis. The expression levels of BNIP3L were analyzed depending on the clinicopathological parameters, such as: sample type (normal, $\mathrm{n}=1 v s$. primary, $\mathrm{n}=104$ vs metastasis, $\mathrm{n}=368$ ), gender (women $v s$. men, $\mathrm{n}=472$ ), age $(<58 v s .>58, \mathrm{n}=464)$, ulceration (no vs. yes, $\mathrm{n}=315$ ), Clark level (I vs. II vs. III-IV vs. V, $\mathrm{n}=323$ ), Breslow depth $(<1$ vs. 1-2 vs. 2.1-4 vs. $>4, \mathrm{n}=361)$, mitotic rate $(0-$ $2 v s .2-3$ vs. $>4, \mathrm{n}=173)$, cancer stage $(0 v s . \mathrm{I}+\mathrm{II} v s . \mathrm{III}+\mathrm{IV}, \mathrm{n}=434)$, M-stage (M0 vs. M1, $\mathrm{n}=444)$, T-stage (T0 vs. T1+T2 vs. T3+T4, $\mathrm{n}=443$ ). Next, from a group of 472 patients, high and low expression subgroups of BNIP3L were selected using the $<25$ and $>25$ percentile as cutoff: i) low $(\mathrm{n}=111)$ and ii) medium with high $(n=351)$, respectively. OS was assessed in these subgroups.

Gene analysis. Using StarBase v3.0 database correlation between BNIP3L and genes connected with autophagy were analyzed in melanoma patients, and next selected genes with $\mathrm{R}$ - square $<-0.2$ and $>0.2$ were compared between the BNIP3L low- and mediumwith high-expressing groups of patients.

Statistical analysis. All statistical analyses were performed using GraphPad Prism 5 (GraphPad, San Diego, CA, USA). The ShapiroWilk normality test, $t$-test, and Mann-Whitney $U$-test or one-way ANOVA performed using Dunn's multiple comparisons test were used for BNIP3L (depending on clinical parameters) and gene expressions (depending on BNIP3L subgroups). For DFS and OS analyses, the Log-Rank (Mantel-Cox) test was used. TCGA data are presented as mean with SEM, and in all analyses, $p 0.05$ was used to determine statistical significance. 
A

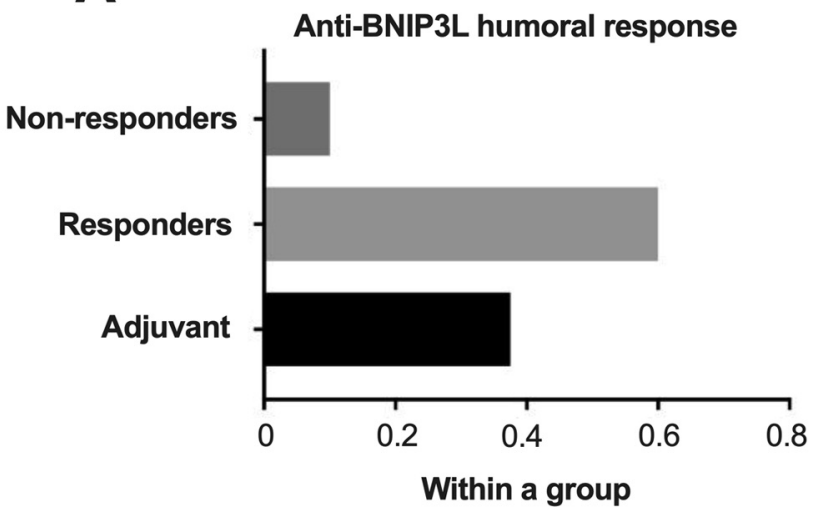

B
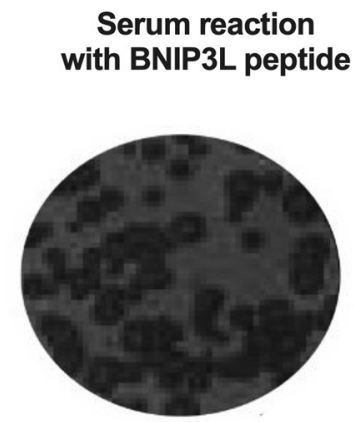

No reaction with BNIP3L peptide

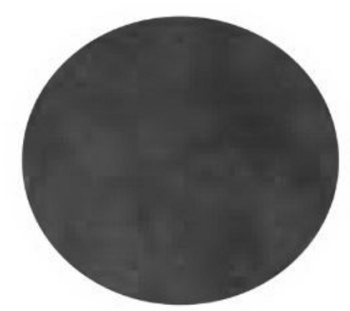

\section{C}

\section{Kaplan-Meier survival plot}

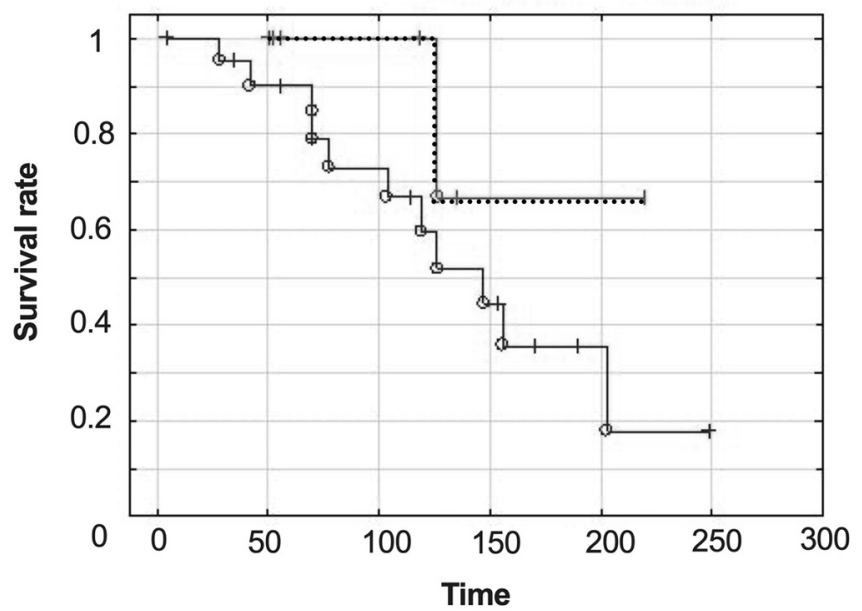

\section{BNIP3L non-responders}

BNIP3L responders

Figure 1. Anti-BNIP3L humoral response in melanoma patients treated with AGI-101H: A) Classification of the patients depending on the response to anti-BNIP3L antibody into the non-responders, responders and adjuvant groups; B) Secondary SEREX blots eliciting seroreactivity with peptide characterized as BNIP3L; C) Progression-free survival probability curves of the two subgroups (AGI-101H responders vs. non-responders) based on the presence of BNIP3L serum response using the Kaplan-Meier survival estimates test.

\section{Results}

AGI-101H treated patients respond to melanoma associated antigens. From the 38 patients analyzed, two patients showed the seroreactivity with the library peptides. These clones were further sequenced. The results were blasted with the mRNA sequences collected in the German Cancer Research Center's HUSAR database. One positive clone was selected and further analyzed. It showed nearly $100 \%$ mRNA homology with a sequence of BNIP3L (BCL-2/adenovirus E1B $19 \mathrm{kd}$-interacting protein-like).

Response to BNIP3L positively correlates with the clinical outcome. In order to evaluate the humoral response to
BNIP3L in other patients, secondary SEREX was carried out. From the 38 patients analyzed, 11 showed reactivity with the clone. This group included 2 of 20 (10\%) patients with disease progression (PD), 6 of $10(60 \%)$ patients with a positive response to the treatment (CR, PR or $\mathrm{SD})$ and $3 / 8$ $(38 \%)$ patients in the adjuvant setting (Figure 1A). Figure 1B presents an example of the visualized serum-clone reaction. The obtained results showed that AGI-101H-responders produced anti-BNIP3L antibodies 6 times more often than non-responders, as measured with odds ratio. The progression risk was 3 times lower in patients who produced anti-BNIP3L antibodies. Increased overall survival was observed in patients with anti-BNIP3L antibodies response. Although these differences were not statistically significant 
due to the small number of individuals, there was a clear tendency towards a positive correlation of anti-BNIP3L response and clinical outcome after AGI-101H treatment as measured by Kaplan-Meier analysis (Figure 1C).

BNIP3L expression is dependent on sample type, stage, and mitotic rate. According to the database (cBioportal and UALCAN), the expression of BNIP3L was significantly upregulated in metastatic melanoma compared to primary samples $(p=6.06 \mathrm{e}-3)$ (Figure 2). Significant differences between expression levels of BNIP3L were observed in patients with Clark level I vs. II $(p=0.0291)$ and mitotic rate $0-2 v s .2-3(p=0.0396)$ and 2-3 vs. $>4(p=0.0376)$ (Figure 3$)$.

BNIP3L expression levels positively correlate with overall survival (OS). Melanoma samples were divided into BNIP3L low, medium and high expression groups using the $<25$ and $>25$ percentile of BNIP3L expression as a cut-off, respectively. Significantly longer OS of BNIP3L medium and high expression group compared to low expression $(p=0.0188)$ was observed with median survival of $98.32 \mathrm{vs}$. 58.48 months (Figure 4).

BNIP3L expression correlates with autophagy-related gene expression. Using the StarBase v3.0 database the correlation between BNIP3L and genes related to autophagy was analyzed in melanoma patients. Genes with R- square $<-0.2$ and $>0.2$ were compared between the BNIP3L low- and medium- with high-expressing groups of patients. There was down-regulation of $A T G 4 D$ gene expression and upregulation of ATG16L1, ATG4A, ATG4C, ATG3, ATG12, ATG5, MAP1LC3B, DRAM1, GABARAPL2, BECN1 expression that are associated with autophagy components in the melanoma patients with medium and high compared to the low expression of BNIP3L (Figure 5). In the group of coregulators of autophagy and apoptosis process in patients with medium and high expression of BNIP3L, downregulation of $C L N 3, A K T 1, B A D, F A D D$ and up-regulation of PIK3CG, TNFSF10 (TRAIL), BNIP3, NFKB1, CASP3, CASP8 (FLICE), FAS (TNFRSF6), MAPK8 (JNK1), EIF2AK3, BENC1 and PRKAA1 (AMPK) were observed compared to the BNIP3L low-expressing group (Figure 6). In the patients with medium and high expression of BNIP3L, down-regulation of CTSD, HGS and up-regulation of UVRAG, ULK2, ESR1 (ER $\alpha$ ), DRAM2 (TMEM77), MAPK14 (p38ALPHA), PIK3R4, PIK3C3 (Vps34) and RPS6KB1, which are associated with autophagy in response to other intracellular signals, were observed. Moreover, in the case of co-regulators of autophagy and cell cycle related genes, CDKN1B (P27KIPl) and RB1, as well as HSP90AAl, chaperone-mediated autophagy gene, up-regulation in patients with medium and high expression of BNIP3L were observed (Figure 7).

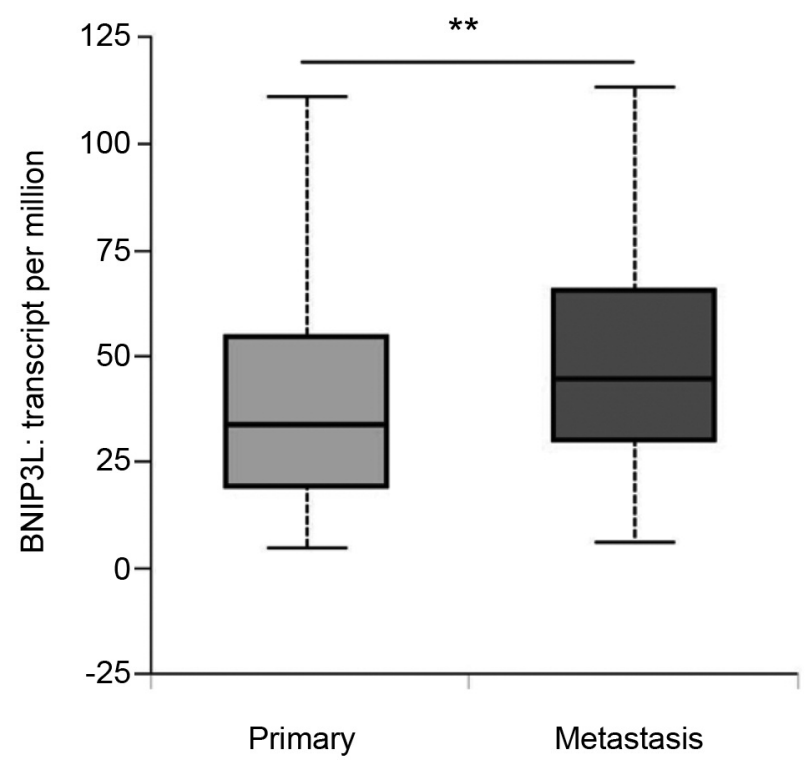

Figure 2. BNIP3L expression in melanoma patients from The Cancer Genome Atlas (TCGA) depending on the sample type (primary: $n=104$ and metastasis: $n=368)$. Box and whiskers with 5-95 percentile, $* * p<0.01$.

\section{Discussion}

So far, no biological marker for monitoring melanoma immunotherapy has been identified. In our study, we found a positive correlation between the production of antibodies to BNIP3L/NIX and the clinical outcome of melanoma patients treated with the whole-cell melanoma vaccine genetically modified to stem cell-like phenotype (AGI$101 \mathrm{H})$. Anti-BNIP3L humoral responses were remarkably enhanced in AGI-101H responders, most likely due to the promotion of the autophagy process, which led to inhibition of melanoma proliferation and extended patient OS as well as DFS. This is the first study reporting the generation of BNIP3L-specific antibodies in patients immunized with whole-cell melanoma vaccine. Our in silico analysis confirmed that increased expression of BNIP3L was also positively correlated with patient OS in melanoma.

BNIP3L plays a crucial role in the clearance of damaged mitochondria in the process of mitochondrial autophagy (mitophagy) in response to the hypoxic conditions (16-18). BNIP3L is induced by tumor suppressor p53 (19-21) and exhibits tumor-suppressing activity itself. The knockdown of BNIP3L promotes tumorigenesis by avoiding p53-dependent apoptosis under hypoxic conditions in a xenograft model of breast cancer (22). BNIP3L also plays a role in immunogenic cancer cell death since BNIP3L-mediated mitophagy promotes the generation of natural killer memory cells (23). Overexpression of BNIP3L in hypoxic conditions is mediated by hypoxia-inducible factor HIF-1 (18). Considering that high 


\section{Clark level}

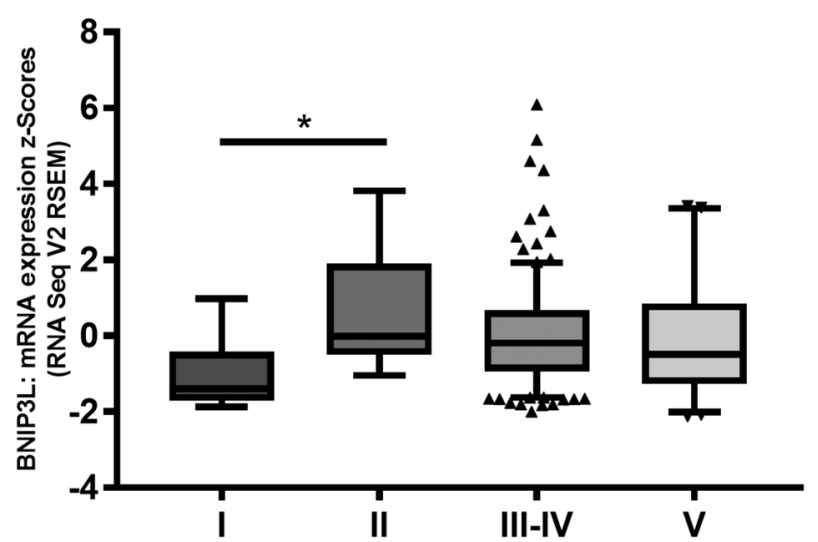

Mitotic rate

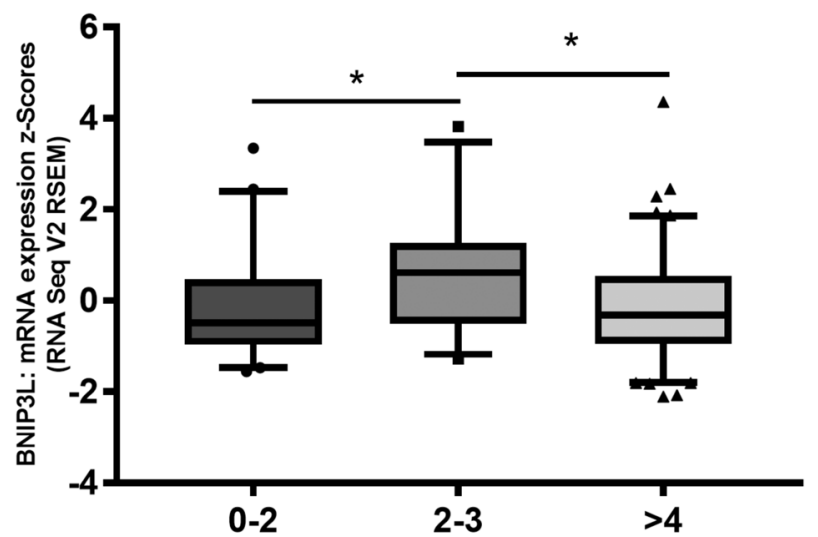

Figure 3. The expression levels of BNIP3L in melanoma are independent of Clark stage and mitotic rate. Box and whiskers with 5-95 percentile; one-way ANOVA obtained using Dunn's multiple comparisons tests; * $p<0.05$.

level of HIF-1 as an indicator of extensive tumor growth (24), overexpression of BNIP3L within the tumor mass may reflect the stage of oncogenic process. The progressive hypoxia leads then to overproduction of BNIP3L. Under the influence of the destroying power of the vaccine, a disintegrated tumor mass releases its components inducing the immune response. The bigger and more damaged a tumor tissue is, the more antigens are released, and the production of antibodies is higher. That scenario would make BNIP3L a prognostic factor for any type of immunotherapy. Also, it defines the level of hypoxia, which indicates the aggressiveness of a tumor, as well the role of autophagy in melanoma, specifically in response to AGI-101H treatment. Since AGI-101H displays CSC phenotype, of particular importance would be the fact that autophagy maintains the pluripotency of cancer stem cells (CSC) and facilitates their survival. Also, it affects epithelial-to-mesenchymal transition (EMT) $(25,26)$. Several markers of autophagy have been identified as potential prognostic biomarkers for melanoma. These include autophagy regulators: LC3, beclin, p62, or AMBRA1. Increased expression of light chain 3 (LC3) has been observed in malignant melanomas compared to benign nevi (27) and was associated with metastasis and poorer outcomes (28). Another study has shown that low beclin 1 expression was associated with high Breslow's depth, high Clark's level, and ulceration (29). Ellis et al. (30) have observed increased expression of autophagy receptor p62 in early AJCC stage melanomas, which was subsequently decreased in advanced metastatic tumors. Decreased or even complete loss of AMBRA1 expression has been observed in the epidermis overlying AJCC stage I melanomas, but not in benign nevi (31) suggesting the prognostic potential of this marker. Another study has shown that loss of Atg7 prevents melanoma development in BrafV600E mutant mice with allelic loss of Pten gene (32).

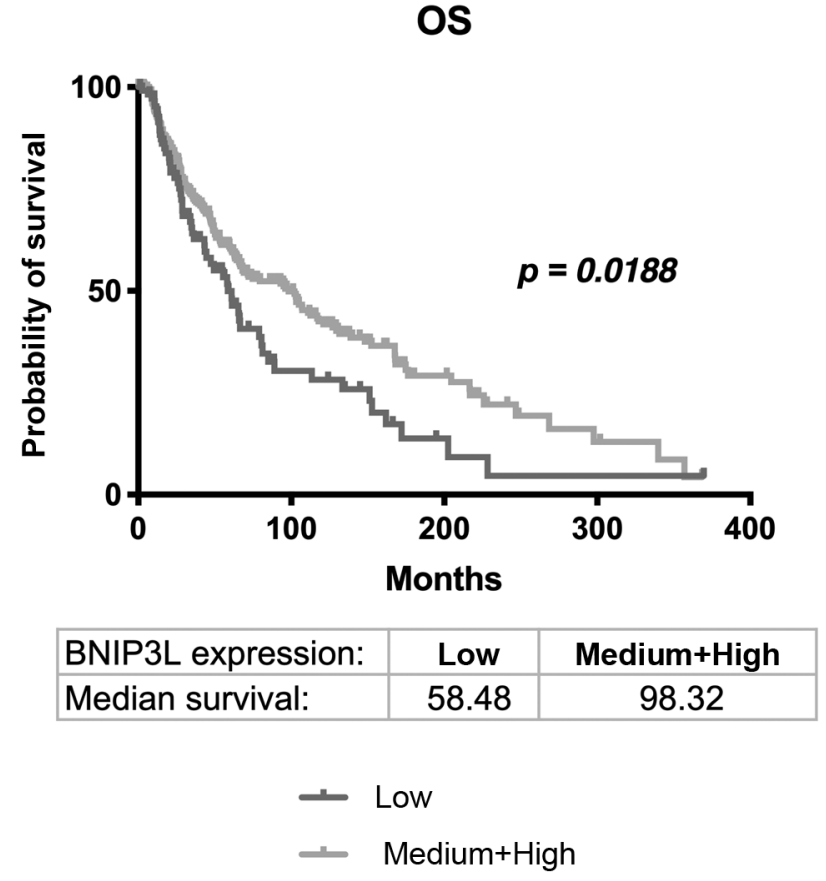

Figure 4. OS in TCGA melanoma patients with low ( $n=111)$ and medium with high ( $n=351)$ expression levels of BNIP3L. Log-rank (Mantel-Cox) test was used for the analysis. $p<0.05$ was considered as significant.

Although in many reports autophagy has been described as cancer progression related factor, there are also a few which show that autophagy displays suppressive functions with a positive impact on clinical outcome (33-35). There exists a general conclusion that autophagy has tumorsuppressive functions in the early stages of cancer and is tumor-promoting in established tumors (36). Interestingly, 


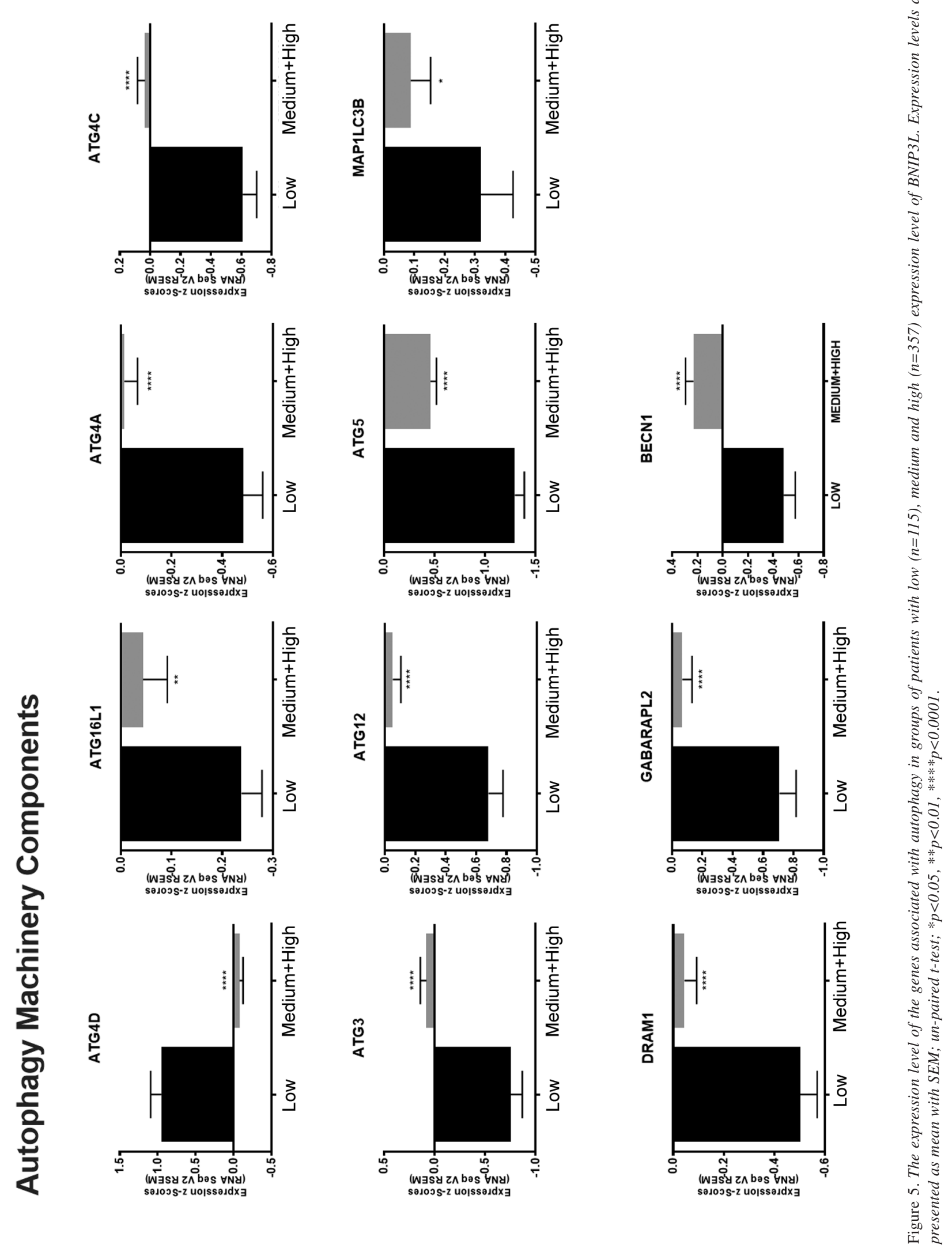



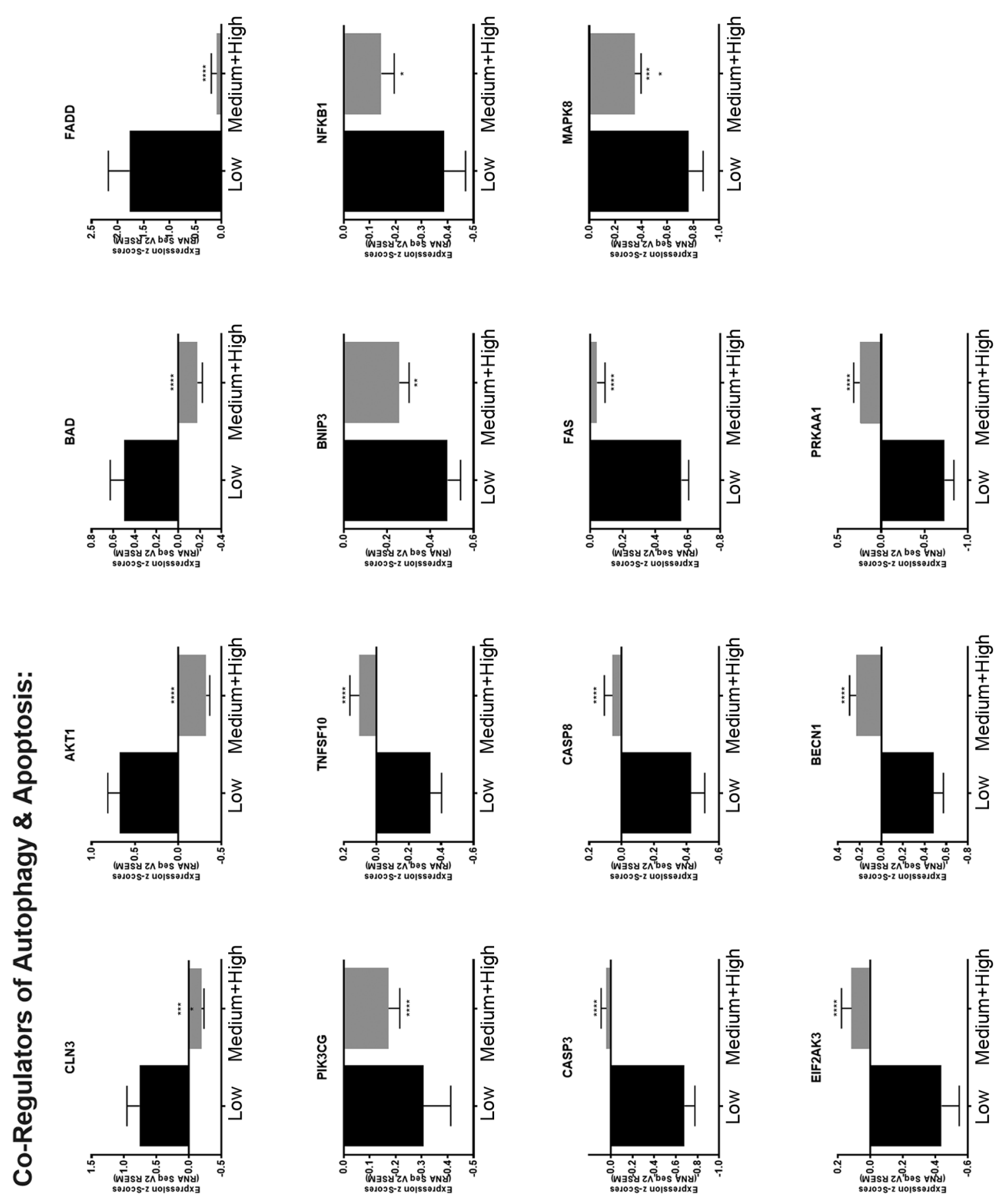

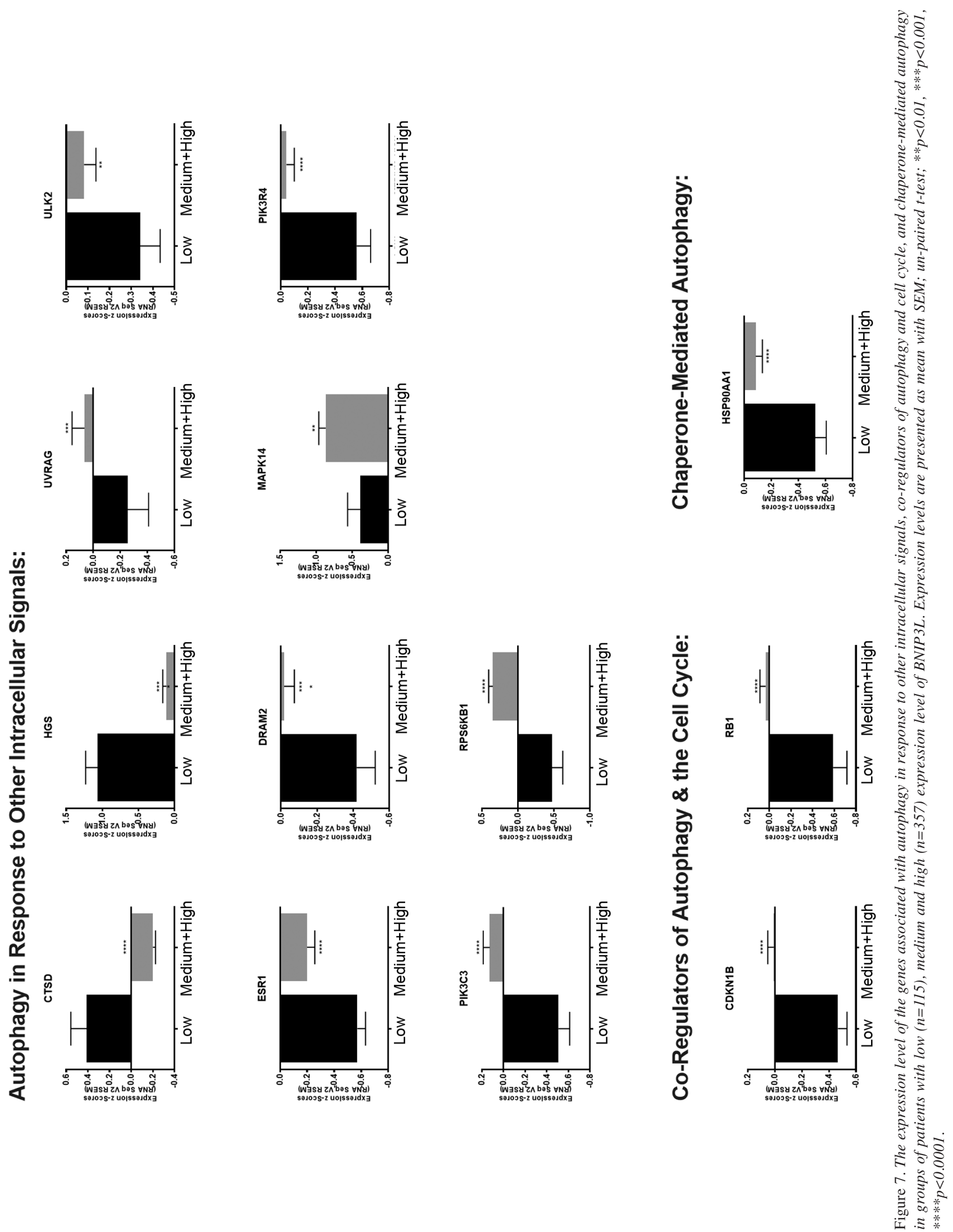
also in metastasis, autophagy reveals its dual role (37) with anti-metastatic capabilities at early stages and pro-metastatic at later stages. Our SEREX analysis showed that AGI-101H stimulates an anti-BNIP3L immune reaction in a group of responders. Thus, we hypothesize that in this group the expression of BNIP3L is enhanced, so does the process of autophagy. However, the level of BNIP3L in tumor samples has not been investigated due to the lack of material. Nonresponders did not produce antibodies to BNIP3L, most likely due to a low level of autophagy. Accordingly, we drew three conclusions: 1) AGI-101H stimulates autophagy which acts as tumor-suppressor, 2) lack of autophagy in advanced melanoma maintains tumor growth, and 3) AGI-101H response is indirectly related with enhanced autophagy, and there are other mechanisms ensuring good clinical outcome.

There is an urgent need to identify predictive/prognostic markers for more efficient, personalized treatment in order to improve clinical outcomes. Here, we demonstrated that BNIP3L is a strong candidate to be considered as a new biomarker for the prognosis of a disease course and melanoma diagnostics. The antibody response in patients receiving specific treatment could determine the effectiveness of therapy.

Since BNIP3L is a critical factor involved in autophagy, which is indicated by enhanced production of BNIP3L antibodies, AGI-101H directs immune response towards autophagy. In view of existing knowledge targeting autophagy may prove to be successful in fighting melanoma.

\section{Conflicts of Interest}

The Authors declare that there are no conflicts of interest regarding this study.

\section{Authors' Contributions}

UK carried out the experiments, analyzed the data and prepared the manuscript. TK performed in silico analyses and prepared the figures. JM prepared the clinical data of analyzed patients. DK and AM revised the manuscript.

\section{Acknowledgements}

The Authors thank Professor Dr. Dirk Schadendorf and Professor Dr. Stefan Eichmuller for enabling the performance of SEREX analyses at the German Cancer Research Center (DKFZ), Heidelberg, Germany. This work was supported by the National Center for Research and Development (Warsaw, Poland) under Grant: INNOMED/6/I/NCBR/2014, "Personalization of melanoma therapeutic vaccination (Per-Mel)".

\section{References}

1 Olszanski AJ: Current and future roles of targeted therapy and immunotherapy in advanced melanoma. J Manag Care Spec Pharm 20(4): 346-356, 2014. PMID: 24684639. DOI: 10.18553/ jmcp.2014.20.4.346
2 Mackiewicz J and Mackiewicz A: BRAF and MEK inhibitors in the era of immunotherapy in melanoma patients. Contemp Oncol (Pozn) 22(1A): 68-72, 2018. PMID: 29628797. DOI: 10.5114/ wo.2018.73890

3 Domingues B, Lopes JM, Soares P and Pópulo H: Melanoma treatment in review. Immunotargets Ther 7: 35-49, 2018. PMID: 29922629. DOI: 10.2147/ITT.S134842

4 Rozeman EA, Dekker TJA, Haanen JBAG and Blank CU: Advanced melanoma: current treatment options, biomarkers, and future perspectives. Am J Clin Dermatol 19(3): 303-317, 2018. PMID: 29164492. DOI: 10.1007/s40257-017-0325-6

5 Yu C, Liu X, Yang J, Zhang M, Jin H, Ma X and Shi H: Combination of immunotherapy with targeted therapy: theory and practice in metastatic melanoma. Front Immunol 10: 990 , 2019. PMID: 31134073. DOI: 10.3389/fimmu.2019.00990.

6 Maleki Vareki S, Garrigós C and Duran I: Biomarkers of response to PD-1/PD-L1 inhibition. Crit Rev Oncol Hematol 116: 116-124, 2017. PMID: 28693793. DOI: 10.1016/j.critrevonc.2017.06.001

7 Long GV, Grob JJ, Nathan P, Ribas A, Robert C, Schadendorf D, Lane SR, Mak C, Legenne P, Flaherty KT and Davies MA: Factors predictive of response, disease progression, and overall survival after dabrafenib and trametinib combination treatment: a pooled analysis of individual patient data from randomised trials. Lancet Oncol 17(12): 1743-1754, 2016. DOI: 10.1016/S14702045(16)30578-28

8 Mackiewicz A, Mackiewicz J, Wysocki PJ, Wiznerowicz M, Kapcinska M, Laciak M, Rose-John S, Izycki S, Burzykowski T and Karczewska-Dzionk A: Long-term survival of high-risk melanoma patients immunized with a Hyper-IL-6-modified allogeneic whole-cell vaccine after complete resection. Expert Opin Investig Drugs 21(6): 773-783, 2012. PMID: 22577889. DOI: $10.1517 / 13543784.2012 .684753$

9 Kwiatkowska-Borowczyk E, Czerwinska P, Mackiewicz J, Gryska K, Kazimierczak U, Tomela K, Przybyla A, Kozlowska AK, Galus L, Kwinta L, Ewelina E, Gabka-Buszek A, Zakowska M, Mackiewicz A: Whole cell melanoma vaccine genetically modified to stem cells like phenotype generates specific immune responses to ALDH1A1 and long-term survival in advanced melanoma patients. Oncoimmunology 7(11): e1509821, 2018. PMID: 30377573. DOI: 10.1080/2162402X.2018.1509821

10 Ozbek S, Peters M, Breuhahn K, Mann A, Blessing M, Fischer M, Schirmacher P, Mackiewicz A and Rose-John S: The designer cytokine hyper-IL-6 mediates growth inhibition and GM-CSFdependent rejection of B16 melanoma cells. Oncogene 20(8): 972-979, 2001. PMID: 11314032. DOI: 10.1038/sj.onc.1204180

11 Mackiewicz J and Mackiewicz A: Design of clinical trials for therapeutic cancer vaccines development. Eur J Pharmacol 625(13): 84-89, 2009. PMID: 30445702. DOI: 10.1016/j.ejphar. 2009.09.069

12 Ehlken H, Schadendorf D and Eichmuller S: Humoral immune response against melanoma antigens induced by vaccination with cytokine gene-modified autologous tumor cells. Int J Cancer 108(2): 307-313, 2004. PMID: 14639620. DOI: 10.1002/ijc.11537

13 The cBioPortal for Cancer Genomics. Available at: http://www.cbioportal.org. [Last accessed May 30, 2020]

14 UALCAN interactive web resource for cancer OMICS data. Available at: http://ualcan.path.uab.edu. [Last accessed May 30, 2020]

15 The encyclopedia of RNA interactomes. Available at: http://starbase.sysu.edu.cn. [Last accessed May 30, 2020] 
16 Schweers RL, Zhang J, Randall MS, Loyd MR, Li W, Dorsey FC, Kundu M, Opferman JT, Cleveland JL, Miller JL and Ney PA: NIX is required for programmed mitochondrial clearance during reticulocyte maturation. Proc Natl Acad Sci USA 104(49): 19500-19505, 2007. PMID: 18048346. DOI: 10.1073/ pnas.0708818104

17 Sandoval H, Thiagarajan P, Dasgupta SK, Schumacher A, Prchal JT, Chen M and Wang J: Essential role for Nix in autophagic maturation of erythroid cells. Nature 454(7201): 232-235, 2008. PMID: 18454133. DOI: 10.1038/nature07006

18 Sowter HM, Ratcliffe PJ, Watson P, Greenberg AH and Harris AL: HIF-1-dependent regulation of hypoxic induction of the cell death factors BNIP3 and NIX in human tumors. Cancer Res 61(18): 6669-6673. PMID: 11559532.

19 Wilfinger N, Austin S, Scheiber-Mojdehkar B, Berger W, Reipert S, Praschberger M, Paur J, Trondl R, Keppler BK, Zielinski CC and Nowikovsky K: Novel p53-dependent anticancer strategy by targeting iron signaling and BNIP3L-induced mitophagy. Oncotarget 7(2): 1242-1261, 2016. PMID: 26517689. DOI: 10.18632 /oncotarget.6233

20 Bruick RK: Expression of the gene encoding the proapoptotic Nip3 protein is induced by hypoxia. Proc Natl Acad Sci USA 97(16): 9082-9087, 2000. PMID: 10922063. DOI: 10.1073/ pnas.97.16.9082

21 Fei P, Wang W, Kim SH, Wang S, Burns TF, Sax JK, Buzzai M, Dicker DT, McKenna WG, Bernhard EJ and El-Deiry WS: Bnip3L is induced by p53 under hypoxia, and its knockdown promotes tumor growth. Cancer Cell 6(6): 597-609, 2004. PMID: 15607964. DOI: 10.1016/j.ccr.2004.10.012

22 Bousquet G, El Bouchtaoui M, Sophie T, Leboeuf C, de Bazelaire C, Ratajczak P, Giacchetti S, de Roquancourt A, Bertheau P, Verneuil L, Feugeas JP, Espié M and Janin A: Targeting autophagic cancer stem-cells to reverse chemoresistance in human triple negative breast cancer. Oncotarget 8(21): 35205-35221, 2017. PMID: 28445132. DOI: 10.18632/oncotarget.16925

23 O'Sullivan TE, Johnson LR, Kang HH and Sun JC: BNIP3- and BNIP3L-mediated mitophagy promotes the generation of natural killer cell memory. Immunity 43(2): 331-342, 2015. PMID: 26253785. DOI: $10.1016 /$ j.immuni.2015.07.012

24 Semenza GL: Defining the role of hypoxia-inducible factor 1 in cancer biology and therapeutics. Oncogene 29(5): 625-634, 2010. PMID: 19946328. DOI: $10.1038 /$ onc. 2009.441

25 Youle RJ and Narendra DP: Mechanisms of mitophagy. Nat Rev Mol Cell Biol 12(1): 9-14, 2011. PMID: 21179058. DOI: $10.1038 / \mathrm{nrm} 3028$

26 Novak I, Kirkin V, McEwan DG, Zhang J, Wild P, Rozenknop A, Rogov V, Löhr F, Popovic D, Occhipinti A, Reichert AS, Terzic J, Dötsch V, Ney PA and Dikic I: Nix is a selective autophagy receptor for mitochondrial clearance. EMBO Rep 11(1): 45-51, 2010. PMID: 30160596. DOI: 10.1038/embor.2009.256

27 Lazova R, Klump V and Pawelek J: Autophagy in cutaneous malignant melanoma. J Cutan Pathol 37(2): 256-268, 2010. PMID: 19615007. DOI: 10.1111/j.1600-0560.2009.01359.x
28 Lazova R, Camp RL, Klump V, Siddiqui SF, Amaravadi RK and Pawelek JM: Punctate LC3B expression is a common feature of solid tumors and associated with proliferation, metastasis, and poor outcome. Clin Cancer Res 18(2): 370-379, 2012. PMID: 22080440. DOI: 10.1158/1078-0432.CCR-11-1282

29 Miracco C, Cevenini G, Franchi A, Luzi P, Cosci E, Mourmouras V, Monciatti I, Mannucci S, Biagioli M, Toscano M, Moretti D, Lio R and Massi D: Beclin 1 and LC3 autophagic gene expression in cutaneous melanocytic lesions. Hum Pathol 41(4): 503-512, 2010. PMID: 20004946. DOI: 10.1016/j.humpath.2009.09.004

30 Ellis RA, Horswell S, Ness T, Lumsdon J, Tooze SA, Kirkham N, Armstrong JL and Lovat PE: Prognostic impact of p62 expression in cutaneous malignant melanoma. J Invest Dermatol 134(5): 1476-1478, 2014. PMID: 24270664. DOI: 10.1038/jid.2013.497

31 Ellis R, Tang D, Nasr B, Greenwood A, McConnell A, Anagnostou ME, Elias M, Verykiou S, Bajwa D, Ewen T, Reynolds NJ, Barrett P, Carling E, Watson G, Armstrong J, Allen AJ, Horswell S, Labus M and Lovat PE: Epidermal autophagy and beclin 1 regulator 1 and loricrin: a paradigm shift in the prognostication and stratification of the American Joint Committee on Cancer stage I melanomas. Br J Dermatol 182(1): 156-165, 2020. PMID: 31056744. DOI: 10.1111/bjd.18086

32 Xie X, Koh JY, Price S, White E and Mehnert JM: Atg7 overcomes senescence and promotes growth of BrafV600Edriven melanoma. Cancer Discov 5(4): 410-423, 2015. PMID: 25673642. DOI: 10.1158/2159-8290.CD-14-1473

33 Luan W, Qian Y, Ni X, Chanda TK, Xia Y, Wang J, Yan Y and $\mathrm{Xu}$ B: Polygonatum odoratum lectin promotes BECN1 expression and induces autophagy in malignant melanoma by regulation of miR1290. Onco Targets Ther 10: 4569-4577, 2017. PMID: 29066911. DOI: 10.2147/OTT.S147406

34 Xiao Y, Diao Q, Liang Y, Peng Y and Zeng K: MicroRNA 241 $5 \mathrm{p}$ promotes malignant melanoma cell autophagy and apoptosis via regulating ubiquitin D. Mol Med Rep 16(6): 8448-8454, 2017. PMID: 28983594. DOI: $10.3892 / \mathrm{mmr} .2017 .7614$

35 Wang WJ, Wang Y, Chen HZ, Xing YZ, Li FW, Zhang Q, Zhou B, Zhang HK, Zhang J, Bian XL, Li L, Liu Y, Bi-xing Zhao, Yan Chen, Rong Wu 2, An-zhong Li, Lu-ming Yao, Chen P, Zhang Y, Tian XY, Beermann F, Wu M, Han J, Huang PQ, Lin T and Wu Q: Orphan nuclear receptor TR3 acts in autophagic cell death via mitochondrial signaling pathway. Nat Chem Biol 10(2): 133-140, 2014. PMID: 24316735. DOI: $10.1038 /$ nchembio. 1406

36 Santana-Codina N, Mancias JD and Kimmelman AC: The role of autophagy in cancer. Ann Rev Cancer Biol 1: 19-39, 2017. PMID: 31119201. DOI: 10.1146/annurev-cancerbio-041816-122338

37 Kenific CM, Thorburn A and Debnath J: Autophagy and metastasis: another double-edged sword. Curr Opin Cell Biol 22(2): 241-245, 2010. PMID: 19945838. DOI: 10.1016/j.ceb.2009.10.008

Received May 10, 2020

Revised May 29, 2020

Accepted May 31, 2020 